\begin{tabular}{c} 
Brazilian Journal \\
of Chemical \\
Engineering \\
\hline
\end{tabular}

ISSN 0104-6632

Printed in Brazil

www.abeq.org.br/bjche

Vol. 32, No. 02, pp. 475 - 487, April - June, 2015

dx.doi.org/10.1590/0104-6632.20150322s00003371

\title{
SELF-SIMILARITY OF VERTICAL BUBBLY JETS
}

\author{
I. E. Lima Neto \\ Assistant Professor, Dept. of Hydraulic and Environmental Engineering, \\ Federal University of Ceará, Fortaleza - CE, Brazil. \\ E-mail: iran@deha.ufc.br
}

(Submitted: March 18, 2014 ; Revised: October 2, 2014 ; Accepted: October 14, 2014)

\begin{abstract}
An integral model for vertical bubbly jets with nearly monodisperse bubble sizes is presented. The model is based on the Gaussian type self-similarity of mean liquid velocity, bubble velocity and void fraction, as well as on functional relationships for initial liquid jet velocity and radius, bubble diameter and relative velocity. Adjusting the model to experimental data available in the literature for a wide range of densimetric Froude numbers provide constant values for the entrainment coefficient, momentum amplification factor, and spreading ratio of the bubble core for different flow conditions. Consistency and sensitivity of key model parameters are also verified. Overall, the deviations between model predictions and axial/radial profiles of mean liquid velocity, bubble velocity and void fraction are lower than about $20 \%$, which suggests that bubbly jets tend to behave as self-preserving shear flows, similarly to single-phase jets and plumes. Furthermore, model simulations indicate a behavior similar to those of single-phase buoyant jets and slurry jets, but some differences with respect to confined bubbly jets are highlighted. This article provides not only a contribution to the problem of self-similarity in two-phase jets, but also a comprehensive model that can be used for analysis of artificial aeration/mixing systems involving bubbly jets.

Keywords: Bubbles; Gaussian profiles; Integral model; Jets; Self-preservation.
\end{abstract}

\section{INTRODUCTION}

Bubbly jets are produced by the injection of gasliquid mixtures into liquids, as shown schematically in Figure 1. Such two-phase flows are encountered in diverse engineering applications, including gas-liquid mass transfer, heat transfer and turbulent mixing in reactors, tanks and water bodies (Sun and Faeth, 1986a; Iguchi et al., 1997; Morchain et al., 2000; Mueller et al., 2002; Lima Neto et al., 2007; Suñol and González-Cinca, 2010; Norman and Revankar, 2011; Lima Neto, 2012b; Zhang and Zhu, 2013).

A number of experimental studies have been conducted to investigate the flow structure of bubbly jets. The first comprehensive investigation on this subject was reported by Sun and Faeth (1986a,b), who studied vertical bubbly jets with gas volume fractions at the nozzle of up to about $9 \%$ and found that mean and turbulent properties of the flow were not significantly affected by the inter-phase transport. Kumar et al. (1989) and Iguchi et al. (1997) conducted experiments on vertical bubbly jets with gas volume fractions of up to 19 and $49 \%$, respectively, and found that increases in this parameter increased slightly the mean liquid velocity, but increased significantly its fluctuating components. Iguchi et al. (1997) also found that radial distributions of mean and turbulent liquid velocities could be fitted to Gaussian distributions. Lima Neto et al. (2008b) investigated vertical bubbly jets with gas volume fractions of up to about $80 \%$ and found that the mean liquid velocity increased significantly with the gas volume fraction. Gaussian type curves were also fitted to the radial distributions of liquid velocity, bubble velocity and void fraction (or gas holdup). In addition, Lima Neto et al. (2008b) obtained slip (or relative) velocities larger than the terminal velocities reported by Clift et al. (1978) for individual

*To whom correspondence should be addressed 
bubbles, and suggested that a nozzle Reynolds number larger than 8.000 is needed to produce relatively small bubbles with approximately uniform diameters. Note that while the studies of Sun and Faeth (1986a,b), Kumar et al. (1989) and Iguchi et al. (1997) were carried out in confined setups (as discussed by Lima Neto et al., 2008c), the study of Lima Neto et al. (2008b) was performed in a large tank, in which the bubbly jets were located far enough from the walls so that they could behave as free jets. Other experimental studies on bubbly jets including the effects of angled-injections, crossflow, collision or periodical excitation of such two-phase flows have also been performed (Varley, 1995; Milenkovic et al., 2007; Lima Neto et al., 2008d; Suñol and González-Cinca, 2010; Zhang and Zhu, 2013). It is interesting to mention that Gaussian profiles of void fraction were still observed in experiments of bubbly jets in crossflow, as pointed out by Zhang and Zhu (2013). Additionally, they found that a nozzle Reynolds number larger than 8.000 is indeed necessary to produce nearly uniform bubble size distributions, as suggested by Lima Neto et al. (2008b). Lima Neto et al. (2008d) obtained the same result for horizontally-injected bubbly jets.

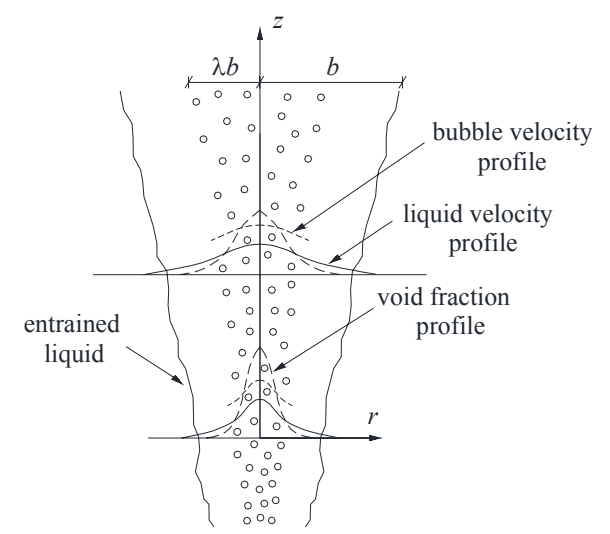

Figure 1: Schematic of a vertical bubbly jet, assuming Gaussian type self-similarity of liquid velocity, bubble velocity and void fraction along the axial direction.

Contrasting with the numerous experimental studies on bubbly jets, theoretical studies on this topic are scarce. Sun and Faeth $(1986 a, b)$ predicted the two-phase flow structure of their dilute vertical bubbly jets (with gas volume fractions at the nozzle of up to about $9 \%$ ) using $k-\varepsilon$ type turbulence models. Reasonably good predictions compared to their experimental data were obtained when bubble slip velocity and bubble-turbulence interactions were con- sidered using random-walk methods. More recently, Lima Neto (2012b) proposed a simple model to predict the mean liquid flow structure of vertical bubbly jets with gas volume fractions of up to $71 \%$, which is based on the Gaussian type self-similarity assumption and integral techniques for single-phase jets and plumes (Morton et al., 1956), but also incorporates the effects of bubble slip velocity and bubble expansion (Cederwall and Ditmars, 1970) and the factor of momentum amplification due to turbulence (Milgram, 1983). Using appropriate conditions for the initial liquid jet velocity and radius and a functional relationship to relate the entrainment coefficient to a densimetric Froude number, Lima Neto's model predicted the liquid velocities induced by bubbly jets. Model simulations plotted together with experimental data for both bubbly jets and bubble plumes also revealed a clear jet/plume transition, suggesting that model simulations could be performed by considering a bubbly jet zone followed by a bubble plume zone. Nevertheless, gas-phase characteristics (bubble velocity and void fraction) were not investigated, since integral models are not expected to accurately predict the net buoyant force acting on similar bubbly flows (i.e., bubble plumes), as pointed out by Socolofsky et al. (2002) and Socolofsky et al. (2008). In fact, even for single-phase jets and plumes, complete self-similarity remains a debatable issue (Wygnanski and Fiedler, 1969; Carazzo et al., 2006; Zhang et al., 2011). Other theoretical studies on bubbly jets including the effects of angled-injections, crossflow and stratification have also been performed (Morchain et al., 2000; Norman and Revankar, 2011).

In the present study, an improved version of the integral model of Lima Neto (2012b) is proposed to predict not only the mean liquid flow induced by vertical bubbly jets, but also the axial and radial distributions of bubble velocity and void fraction. Parameterization and optimization of key model parameters, as well as model comparison with experimental data available in the literature, are then conducted in order to investigate whether bubbly jets tend to complete or violate self-similarity, considering gas volume fractions at the source ranging from very low to high. In addition, model simulations are compared to the behavior of single-phase buoyant jets, confined bubbly jets and slurry jets and the differences are highlighted. This paper provides not only a contribution to the problem of self-similarity in two-phase shear flows, but also a generalized model that can be used in practical applications involving vertical bubbly jets in still liquids. 


\section{MODEL FORMULATION, PARAMETERIZATION AND OPTIMIZATION}

The model proposed in this study assumes that vertical bubbly jets exhibit self-similarity, as usually done for single-phase jets and plumes (Zhang et al., 2011). Thus, in order to simplify the relationships for the variation of the two-phase flow properties of the bubbly jets along the axial direction $z$, the distributions of liquid velocity $(u)$, bubble velocity $(w)$ and void fraction $(C)$ along the radial direction $r$ are assumed to be Gaussian (see Figure 1):

$$
\begin{aligned}
& u(r, z)=u_{c}(z) e^{-r^{2} / b^{2}} \\
& w(r, z)=u_{c}(z) e^{-r^{2} / b^{2}}+u_{s} \\
& C(r, z)=C_{c}(z) e^{-r^{2} /(\lambda b)^{2}}
\end{aligned}
$$

in which $u_{c}, w_{c}$ and $C_{c}$ are respectively the liquid velocity, bubble velocity, and void fraction at the bubbly jet centerline, $b$ is a measure of the liquid jet radius where the liquid velocity $u$ is $e^{-1}=37 \%$ of the centerline value, $u_{s}$ is the bubble slip velocity (assumed constant for monodisperse bubble diameters), and $\lambda$ is the spreading ratio of the bubble core radius relative to the liquid jet radius.

Because the distributions of $u, w$ and $C$ are described by Eqs. (1) - (3), state variables can be integrated over the radial direction $r$ to obtain integral fluxes of volume, momentum and buoyancy that are functions only of the axial direction $z$, reducing the problem to one dimension. Hence, the following equations are obtained by invoking conservation of volume (using the entrainment hypothesis closure), conservation of momentum (equating the spatial rate of change of momentum flux to the total buoyancy force per unit height of the jet), and conservation of the dispersed phase buoyancy (using the ideal gas law together with adiabatic expansion):

$$
\begin{aligned}
& \frac{d}{d z}\left(u_{c} \pi b^{2}\right)=2 \pi b u_{c} \alpha \\
& \frac{d}{d z}\left(\frac{\gamma}{2} \rho_{l} u_{c}^{2} \pi b^{2}\right)=\pi \lambda^{2} b^{2} C_{c} g\left(\rho_{l}-\rho_{g}\right) \\
& \frac{d}{d z}\left[\pi \lambda^{2} b^{2} C_{c} g\left(\rho_{l}-\rho_{g}\right)\left(\frac{u_{c}}{1+\lambda^{2}}+u_{s}\right)\right] \\
& =\frac{d}{d z}\left[\frac{\left(\rho_{l}-\rho_{g}\right) Q_{g a} H_{a}}{H-z}\right]
\end{aligned}
$$

in which $\alpha$ is the entrainment coefficient, $\gamma$ is the factor of momentum amplification due to turbulence, $\rho_{l}$ and $\rho_{g}$ are the liquid and gas density, respectively, $Q_{\mathrm{ga}}$ is the gas volume flow rate at atmospheric pressure, $H_{\mathrm{a}}$ is the atmospheric pressure head, and $H$ is the static pressure head at the nozzle. Eq. (4) is the same proposed by Lima Neto (2012b) to evaluate the axial variation of the liquid volume flux induced by bubbly jets. Eq. (5) is modified from the corresponding equation of Lima Neto (2012b) in order to express the axial variation of the momentum flux in terms of the centerline void fraction $C_{c}$. Eq. (6) is included here to allow the evaluation of the buoyancy flux (or the centerline void fraction $C_{c}$ ) along the axial direction. Therefore, the integral model described herein is based on a system of three conservation equations in the form of ordinary differential equations, in which the variables to be solved for are $u_{c}, b$, and $C_{c}$. Observe that gas-liquid mass transfer is neglected in the present study, but may be included for deep water applications (see Wüest et al., 1992). Moreover, for such conditions, a jet/plume transition may also exist, so that the model must be modified in order to incorporate this effect (see Lima Neto, 2012b).

The bubble slip velocity $u_{\mathrm{s}}$ can be obtained using the following relationship, which was adjusted herein with the experimental data of Lima Neto et al. (2008a,b,d) for mean bubble diameters $d_{\mathrm{b}}$ ranging from about 1 to $4 \mathrm{~mm}$ :

$u_{s}=4.3 d_{b}+34.6$

where $u_{\mathrm{s}}$ is given in $\mathrm{cm} / \mathrm{s}$ and $d_{\mathrm{b}}$ is given in $\mathrm{mm}$. Eq. (7) predicts the experimental data of Lima Neto et al. (2008a,b,d) with deviations of up to about $25 \%$. Therefore, this equation will be used in the present model, rather than using an average value of $u_{\mathrm{s}}$ for all simulations (Lima Neto, 2012b). Observe that Eq. (7) provides bubble slip velocities ranging from about 1.3 to 2.1 times higher than the corresponding terminal velocities reported by Clift et al. (1978) for individual bubbles.

On the other hand, the mean bubble diameter $d_{\mathrm{b}}$ can be obtained by adjusting the following relationship to the experimental data of Sun and Faeth (1986a,b), Iguchi et al. (1997), Lima Neto et al. (2008b,d) and Zhang and Zhu (2013):

$\frac{d_{b}}{\delta}=0.3 \times 10^{4}\left(\frac{C_{o}}{\operatorname{Re}}\right)+0.1$

In Eq. (8), $\delta$ is a length scale already used for bubble plumes (Lima Neto, 2012a), which is given 
by $\delta=\left(Q_{g o}^{2} / g\right)^{1 / 5}$, while $C_{\mathrm{o}}$ is the gas volume fraction at the nozzle and Re is the Reynolds number based on the superficial liquid velocity:

$$
\begin{aligned}
C_{o} & =\frac{Q_{g o}}{Q_{g o}+Q_{l o}} \\
\operatorname{Re} & =\frac{4 Q_{l o}}{\pi d v_{l}}
\end{aligned}
$$

where $Q_{g_{0}}$ and $Q_{l o}$ are the gas and liquid volume flow rates at the nozzle, respectively, $d$ is the nozzle diameter and $v_{1}$ is the liquid kinematic viscosity.

Eq. (8) predicts the experimental data of Sun and Faeth (1986a,b), Iguchi et al. (1997), Lima Neto et al. (2008b,d) and Zhang and Zhu (2013) with deviations of up to about $20 \%$ and thus will be used in the present model. Furthermore, this equation can also be used in bubbly jet studies considering gas-liquid mass transfer effects.

It is relevant to stress that the above relationships [Eqs. (7) and (8)] were obtained for nearly uniform bubble size distributions. As already mentioned, this condition was attained for $\mathrm{Re}>8.000$ (see Lima Neto et al., 2008b,d; Zhang and Zhu, 2013). Note that the data of Kumar et al. (1989) was not considered here because most of their tests were conducted for $\operatorname{Re}<8.000$ and they had to use a screen assembly before the nozzle to break large bubbles into smaller bubbles and produce approximately uniform sizes.

Assuming that uniform bubble sizes result from the occurrence of the so-called bubbly flow regime at the source, the drift flux model of Zuber and Findlay (1965) seems appropriate to evaluate the liquid velocity at the nozzle exit, as already suggested by Iguchi et al. (1997):

$u_{o}=\frac{\left(1-\theta C_{o}\right) V_{T}-C_{o} V_{G}}{\left(1-C_{o}\right)}$

where $\theta$ is a distribution parameter, $V_{T}$ is the average volumetric flux density, and $V_{G}$ is the drift velocity, which are given by the following expressions:

$$
\begin{aligned}
& \theta=1.2-0.2\left(\frac{\rho_{g}}{\rho_{l}}\right)^{1 / 2} \\
& V_{T}=\frac{Q_{l o}+Q_{g o}}{\pi d^{2} / 4}
\end{aligned}
$$

$V_{G}=\sqrt{2}\left[\frac{\sigma g\left(\rho_{l}-\rho_{g}\right)}{\rho_{l}^{2}}\right]^{1 / 4}$

in which $\sigma$ is the liquid surface tension.

Therefore, Eq. (11) will be used herein to evaluate the liquid velocity at the nozzle exit. For the sake of simplicity, the model of Lima Neto (2012b) used Eq. (13) to estimate this velocity, while the experimental study of Lima Neto et al. (2008b) considered the superficial liquid velocity [obtained by setting $Q_{g_{0}}$ to zero in Eq. (13)] to fit their empirical correlations. Such alternative approaches will also be tested in the present study.

Instead of using corrections for the virtual origin of the flow, as often done for bubble plumes (Lima Neto, 2012a), the liquid jet radius at the nozzle exit was simply taken as equal to the nozzle diameter:

$b_{o}=d$

This assumption is supported by experimental observations of Lima Neto et al. (2008b) and was used by Lima Neto (2012b) to predict the liquid volume flux induced by bubbly jets.

Finally, the three remaining parameters to be determined from the governing equations [Eqs. (4) (6)] are the spreading ratio of the bubble core $\lambda$, entrainment coefficient $\alpha$, and momentum amplification factor $\gamma$. Since the model described here assumes that bubbly jets exhibit self-similarity, the abovementioned parameters must be considered constants. Hence, provided these constants are known, the system of governing equations can be solved simultaneously using an appropriate numerical scheme $\left(4^{\text {th }}\right.$ order Runge-Kutta) to yield values of $u_{c}, b$ and $C_{c}$ along the axial direction $z$. Furthermore, the distributions of liquid velocity $u$, bubble velocity $w$ and void fraction $C$ along the radial direction $r$ can be obtained from Eqs. (1) - (3). Note that, for all the numerical simulations, small values of $d z$ of up to about $1 \%$ of the water depth $H$ were used. These values were fine enough to yield accurate results, with deviations of less than $1 \%$ from those obtained with finer step sizes.

Integral models for bubble plumes usually take $\lambda$ as a constant, but $\alpha$ and $\gamma$ are normally described as functions of the flow conditions and/or by the axial distance from the source (Milgram, 1983; Socolofsky et al., 2002; Lima Neto, 2012a). On the other hand, the integral model for bubbly jets proposed by Lima Neto (2012b) used average values for $\lambda$ and $\gamma$ and a functional relationship based on a densimetric Froude 
number to evaluate $\alpha$ for each flow condition. In the present study, in order to reach a complete selfsimilarity of the flow, the parameters $\lambda, \alpha$, and $\gamma$ will be fitted to experimental data of Lima Neto et al. (2008b), which included tests with densimetric Froude numbers at the nozzle exit ranging from 5.4 to 47.5 (see Expt. 1-15 in Table 1). Note that the densimetric Froude number used in this study differs from that of Lima Neto (2012b) and is given by the following expression:

$$
F r=\frac{u_{o}}{\sqrt{d g\left(\rho_{l}-\rho_{m}\right) / \rho_{l}}}
$$

where the mixture density at the nozzle $\rho_{m}$ is defined as $\rho_{m}=\left[\left(1-C_{o}\right) \rho_{l}+C_{o} \rho_{g}\right]$. Since Eq. (16) accounts for both momentum and buoyancy forces at the source, including the effect of $\rho_{m}$ for each flow condition, it is more appropriate than that of Lima Neto (2012b) for comparison of the behavior of bubbly jets with single-phase buoyant jets, which will be done in the next section. For the single-phase case, $\rho_{m}$ is simply taken as the liquid jet density at the source.

Therefore, the constant values for the spreading ratio of the bubble core $\lambda$, entrainment coefficient $\alpha$, and momentum amplification factor $\gamma$ were optimized by solving the following objective function used to minimize the deviations between the present integral model predictions and the experimental data of Lima Neto et al. (2008b):

Minimize: $\sum_{i=1}^{n} \sum_{j=1}^{m}\left(\chi_{i}-\chi_{i}^{\prime}\right)^{2}$

by varying $\alpha, \gamma$ and $\lambda$, subject to the following constraints within the ranges reported by Milgram (1983), Socolofsky et al. (2002), Lima Neto et al. (2008a,b,c,d) and Lima Neto (2012a,b) for bubble plumes and bubbly jets:

$0.040 \leq a \leq 0.120$

$$
1.0 \leq g \leq 3.0
$$

$$
0.4 \leq l \leq 1.0
$$

In Eq. (17), $n$ and $m$ correspond respectively to the number of flow conditions $(n=15)$ studied by Lima Neto et al. (2008b) (see Table 1) and the number of data points $(m=8)$ for each experimental condition. The parameters $\chi_{i}$ and $\chi_{i}^{\prime}$ represent respectively the model predictions and experimental data of mean liquid velocity (4 data points) and void fraction (4 data points), for each experimental condition (total of 15 experiments $=120$ data points). The objective function was used to minimize the sum of the deviations between $\chi_{i}$ and $\chi_{i}^{\prime}$, i.e., the sum of 120 deviations between model predictions and experiments.

\begin{tabular}{|c|c|c|c|c|c|c|c|c|c|}
\hline Expt. & Reference & $\begin{array}{c}\mathbf{H} \\
(\mathbf{m})\end{array}$ & $\begin{array}{c}\mathbf{L} \\
(\mathbf{m})^{*}\end{array}$ & $\begin{array}{c}\mathbf{d} \\
(\mathbf{m m})\end{array}$ & $\begin{array}{c}\text { Qgo } \\
(1 / \mathrm{min})\end{array}$ & $\begin{array}{c}\text { Qlo } \\
(\mathbf{l} / \mathrm{min})\end{array}$ & $\begin{array}{c}\text { Co } \\
(\%) \\
\end{array}$ & $\operatorname{Re}$ & $\mathbf{F r}$ \\
\hline 1 & & & & 13.5 & 3.00 & 7.00 & 30.0 & 11003 & 5.4 \\
\hline 2 & & & & 9.0 & 5.00 & 3.50 & 58.8 & 8252 & 7.1 \\
\hline 3 & & & & 9.0 & 2.00 & 5.00 & 28.6 & 11789 & 10.7 \\
\hline 4 & & & & 6.0 & 3.00 & 3.00 & 50.0 & 10610 & 16.6 \\
\hline 5 & & & & 6.0 & 5.00 & 3.00 & 62.5 & 10610 & 16.7 \\
\hline 6 & & & & 6.0 & 5.00 & 4.00 & 55.6 & 14147 & 22.3 \\
\hline 7 & & & & 6.0 & 5.00 & 5.00 & 50.0 & 17684 & 27.7 \\
\hline 8 & Lima Neto et al. (2008b) & 0.8 & 1.2 & 6.0 & 4.00 & 5.00 & 44.4 & 17684 & 27.8 \\
\hline 9 & & & & 9.0 & 0.40 & 7.00 & 5.4 & 16505 & 27.8 \\
\hline 10 & & & & 6.0 & 3.00 & 5.00 & 37.5 & 17684 & 28.1 \\
\hline 11 & & & & 4.0 & 5.00 & 2.00 & 71.4 & 10610 & 28.7 \\
\hline 12 & & & & 6.0 & 2.00 & 5.00 & 28.6 & 17684 & 29.4 \\
\hline 13 & & & & 4.0 & 1.00 & 2.00 & 33.3 & 10610 & 31.5 \\
\hline 14 & & & & 6.0 & 1.00 & 5.00 & 16.7 & 17684 & 34.4 \\
\hline 15 & & & & 6.0 & 0.40 & 5.00 & 7.4 & 17684 & 47.5 \\
\hline 16 & & & & 5.1 & 0.20 & 2.00 & 9.1 & 8355 & 26.4 \\
\hline 17 & Sun and Faeth $(1986 a, b)$ & 0.9 & 0.4 & 5.1 & 0.10 & 2.00 & 4.8 & 8355 & 34.8 \\
\hline 18 & & & & 5.1 & 0.05 & 2.00 & 2.4 & 8355 & 47.4 \\
\hline 19 & & & & 5.0 & 2.40 & 2.50 & 49.0 & 10610 & 22.1 \\
\hline 20 & Iguchi et al. (1997) & 0.4 & 0.2 & 5.0 & 1.20 & 2.50 & 32.4 & 10610 & 22.8 \\
\hline 21 & & & & 5.0 & 0.60 & 5.00 & 10.7 & 21221 & 63.7 \\
\hline
\end{tabular}

Table 1: Experimental conditions of vertical bubbly jets used for comparison with the present model simulations.

$* \mathrm{~L}$ is the width (or diameter) of the tank 
Observe that this data only included values of mean liquid velocity and void fraction, since bubble velocity can be obtained directly from liquid velocity by using Eqs. (2) and (7).

The next section shows model predictions of the mean liquid velocity, bubble velocity and void fraction using the optimized values of $\alpha, \gamma$ and $\lambda$. The impact of different combinations of $\alpha, \gamma$, and $\lambda$, as well as of different approaches for evaluation of the liquid velocity at the nozzle exit and the bubble relative velocity are also investigated. Model predictions are compared not only with the data of liquid velocity and void fraction used to obtain the fitting parameters $(\alpha, \gamma$ and $\lambda)$, but also with independent data of Lima Neto et al. (2008b) for bubble velocity, Iguchi et al. (1997) for liquid velocity, and Sun and Faeth $(1986 a, b)$ for both liquid and bubble velocity. Again, the experimental results of Kumar et al. (1989) were not used for comparison as their study focused on the turbulence near the nozzle exit of very confined bubbly jets. Finally, model simulations are compared to the behavior of single-phase buoyant jets, confined bubbly jets, and slurry jets.

\section{RESULTS AND DISCUSSION}

Optimized values of the entrainment coefficient ( $\alpha=0.077)$, momentum amplification factor $(\gamma=$ $1.0)$ and spreading ratio of the bubble core $(\lambda=0.6)$ were obtained by solving Eq. (17). As an example, Table 2 shows the impact of ten different combinations of $\alpha, \gamma$, and $\lambda$ on the standard deviations (STD) between model predictions and experimental data of mean liquid velocity $(u)$ and void fraction $(C)$ given by Lima Neto et al. (2008b), which included tests with densimetric Froude numbers at the nozzle ranging from 5.4 to 47.5 (see Expt. 1-15 in Table 1). Model predictions using the optimized values resulted in standard deviations of 10 and $18 \%$ as compared to experimental data of liquid velocity and void fraction, respectively. This also resulted in coefficients of determination higher than 0.95 for all the cases depicted in Figures 2 and 3. Note that the normalized radial profiles of $u_{c} / u_{o}$ and $C_{c} / C_{o}$ cover a range of $z / d$ from about 30 to 120 . These give credence to the model and support the hypothesis that bubbly jets behave as self-preserving shear flows following approximately the Gaussian type self-similarity, as already observed for single-phase jets and plumes (Wygnanski and Fiedler, 1969; Zhang et al., 2011).
Table 2: Impact of different combinations of the entrainment coefficient $(\alpha)$, momentum amplification factor $(\gamma)$, and spreading ratio of the bubble core $(\lambda)$ on the standard deviations between model predictions and experimental data of mean liquid velocity $(u)$ and void fraction. $(C)$.

\begin{tabular}{|c|c|c|c|c|c|c|}
\hline Interaction & $\boldsymbol{\alpha}$ & $\boldsymbol{\gamma}$ & $\boldsymbol{\lambda}$ & $\begin{array}{c}\text { STD of } \\
\mathbf{u}\end{array}$ & $\begin{array}{c}\text { STD of } \\
\mathbf{C}\end{array}$ & $\begin{array}{c}\text { Average } \\
\text { STD }\end{array}$ \\
\hline 1 & 0.040 & 1.0 & 0.4 & $39 \%$ & $73 \%$ & $56 \%$ \\
2 & 0.120 & 3.0 & 1.0 & $29 \%$ & $64 \%$ & $47 \%$ \\
3 & 0.040 & 2.0 & 0.7 & $37 \%$ & $47 \%$ & $42 \%$ \\
4 & 0.120 & 2.0 & 0.7 & $28 \%$ & $50 \%$ & $39 \%$ \\
5 & 0.080 & 2.0 & 1.0 & $13 \%$ & $50 \%$ & $32 \%$ \\
6 & 0.080 & 2.0 & 0.4 & $15 \%$ & $44 \%$ & $30 \%$ \\
7 & 0.080 & 3.0 & 0.7 & $16 \%$ & $30 \%$ & $23 \%$ \\
8 & 0.080 & 2.0 & 0.7 & $14 \%$ & $29 \%$ & $22 \%$ \\
9 & 0.080 & 1.0 & 0.7 & $10 \%$ & $28 \%$ & $19 \%$ \\
10 & 0.077 & 1.0 & 0.6 & $10 \%$ & $18 \%$ & $14 \%$ \\
\hline
\end{tabular}

The value of $\alpha=0.077$ is within the values reported by Zhang et al. (2011) for single-phase jets $(\alpha=0.057)$ and single-phase plumes $(\alpha=0.088)$. Consistently, this value is slightly higher than $\alpha=$ 0.075 , which was estimated by using the analytical solution for single-phase buoyant jets given by Zhang et al. (2011) and the present conditions of Fr. Therefore, it can be inferred that bubbly jets induce slightly higher entrainment than single-phase buoyant jets, probably because of the additional entrainment into the wakes of the bubbles, as already discussed by Lima Neto et al. (2008b). The present model simulations were very sensitive to $\alpha$. Increasing this parameter to $\alpha=0.120$ (which means more entrainment of ambient liquid) resulted in liquid velocities and void fractions of up to about $40 \%$ and $60 \%$ smaller than those shown in Figures 2 and 3, respectively. On the other hand, decreasing this parameter to $\alpha=0.040$ (which means less entrainment of ambient liquid) resulted in liquid velocities and void fractions of up to about 2 and 3 times higher than those shown in Figures 2 and 3, respectively. This also indicates that entrainment variation had a higher impact on the void fraction than on the liquid velocity.

The value of $\gamma=1.0$ implies that the turbulent transport may be negligible as compared to the total mean flow transport. As a matter of fact, the experimental results of Wygnanski and Fiedler (1969) for single-phase jets and of Sun and Faeth (1986a,b), Kumar et al. (1989) and Iguchi et al. (1997) for bubbly jets indicate values of $\gamma$ of up to about 1.2. Note that the results for bubbly jets are limited to smallscale and confined setups, where the effects of turbulence are expected to be higher than for free or unconfined bubbly jets, as in the present case. 


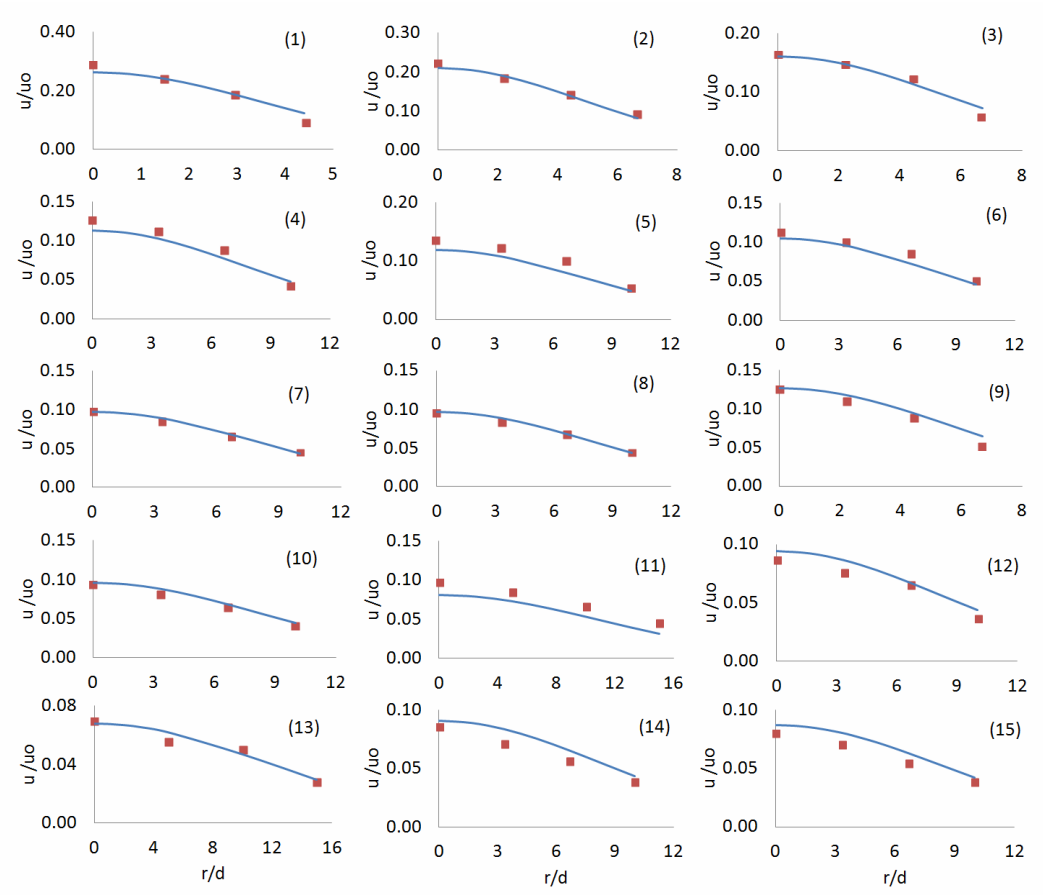

Figure 2: Comparison between model predictions and experimental data of mean liquid velocity given by Lima Neto et al. (2008b). The numbers (1) to (15) correspond to the experimental conditions shown in Table 1 . The normalized radial profiles of $u / u_{o}$ cover a range of $z / d$ from about 30 to 120 .
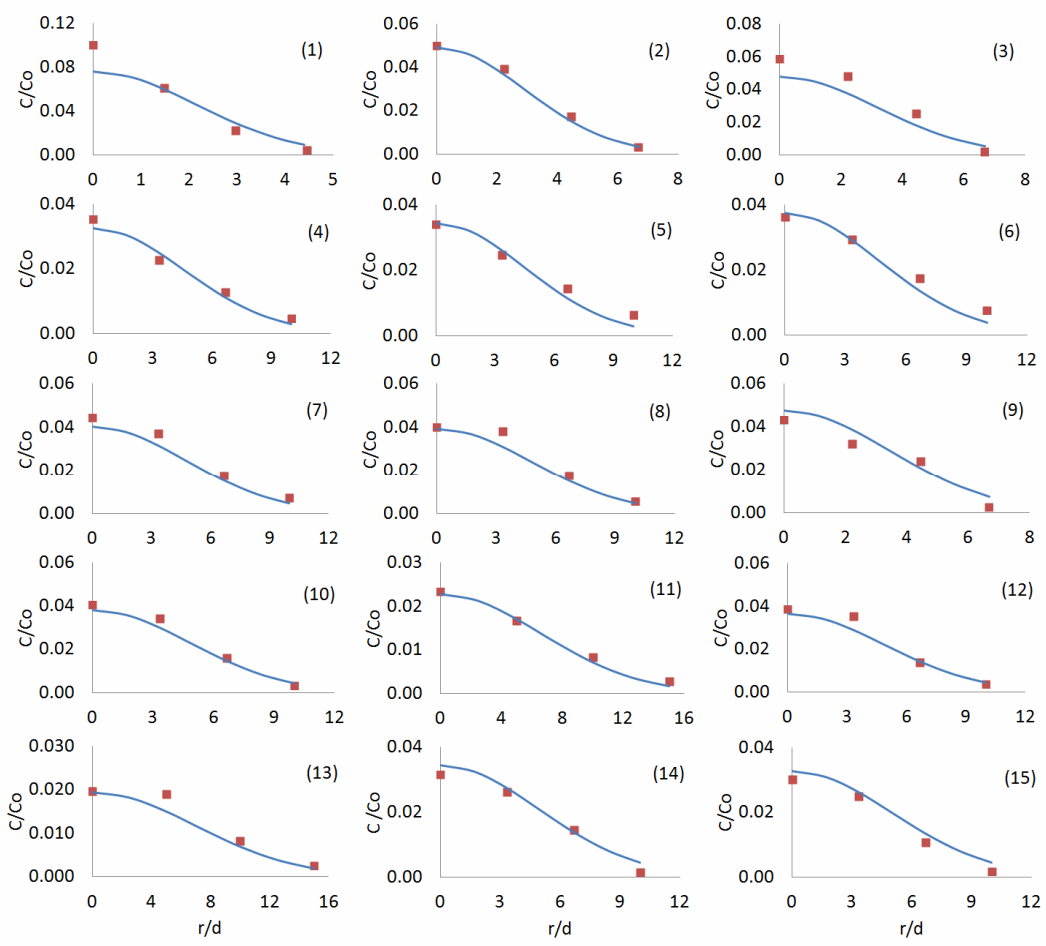

Figure 3: Comparison between model predictions and experimental data of void fraction given by Lima Neto et al. (2008b). The numbers (1) to (15) correspond to the experimental conditions shown in Table 1. The normalized radial profiles of $C / C_{o}$ cover a range of $z / d$ from about 30 to 120 . 
Therefore, the fitted value of $\gamma=1.0$ seems reasonable. The simulations were less sensitive to $\gamma$ than to $\alpha$. Increasing this parameter to $\gamma=3.0$ (which means more turbulence) resulted in liquid velocities and void fractions of up to about $30 \%$ and $10 \%$ smaller than those indicated in Figures 2 and 3, respectively. It is also interesting to observe that turbulence consistently had a higher impact on the liquid velocity than on the void fraction.

The value of $\lambda=0.6$ is smaller than the typical values of 0.7 and 0.8 previously used by Lima Neto (2012b) and Lima Neto (2012a) to predict the liquid volume fluxes induced by bubbly jets and bubble plumes, respectively. The impact of $\lambda$ on the present model simulations of liquid velocity was very small, yielding variations of less than $3 \%$ of the results shown in Figure 2. Nonetheless, $\lambda$ had a significant impact on void fraction. Increasing this parameter to $\lambda=1.0$ (which means more spreading of the bubble core) resulted in void fractions of up to about $70 \%$ smaller than those shown in Figure 3. On the other hand, decreasing this parameter to $\lambda=0.4$ (which means less spreading of the bubble core) resulted in void fractions of up to about 2 times higher than those shown in Figure 3. Therefore, the spreading ratio variation had a negligible impact on the liquid velocity but a significant impact on the void fraction. This is consistent with the results shown in Table 2 (see interactions 9 and 10), in which a relatively small drop in $\lambda$ from 0.7 (used by Lima Neto, 2012b) to 0.6 (used herein) yielded an improvement in the prediction of void fraction from 28 to $19 \%$.

Alternatively, the solution of Eq. (17) using Eq. (13) yielded the following values: $\alpha=0.090, \gamma=1.0$, and $\lambda=0.5$, which are close to those obtained by using Eq. (11) instead. Model predictions using these values resulted in standard deviations of 11 and $20 \%$ as compared to experimental data of liquid velocity and void fraction, respectively, as well as in coefficients of determination higher than 0.94 for all the cases shown in Figures 2 and 3. This indicates that the use of the drift flux model to predict the two-phase flow structure of vertical bubbly jets provides slightly better results than the use of the average volumetric flux density as an approximation for the initial liquid velocity. On the other hand, the use of the superficial liquid velocity instead of Eq. (11) or Eq. (13) resulted in much larger deviations $(>100 \%)$ for some test conditions. Therefore, only the simulations obtained with Eq. (11) will be presented here.

Figure 4 shows a comparison between model predictions and experimental data of bubble velocity of Lima Neto et al. (2008b). The standard deviations were lower than $10 \%$ and the coefficient of determination was higher than 0.94 for all the cases (Expt. 115 in Table 1).
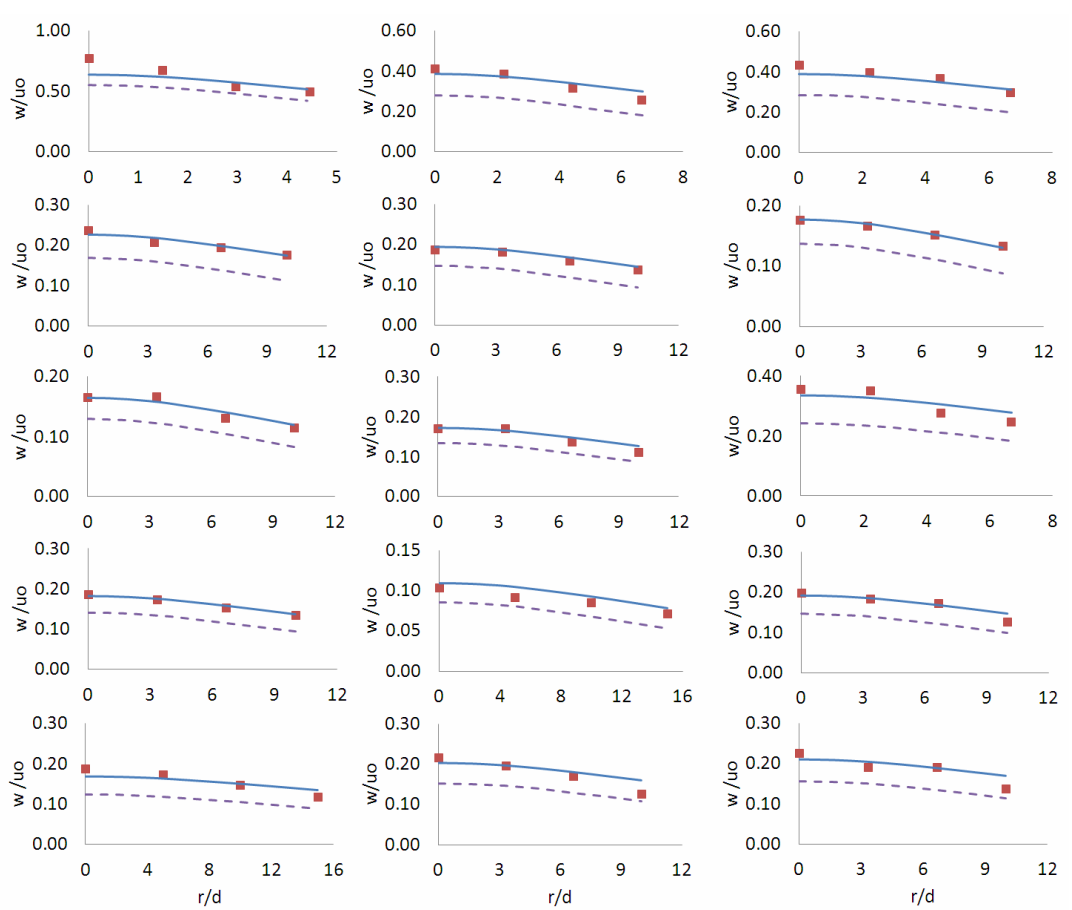

Figure 4: Comparison between model predictions and experimental data of bubble velocity given by Lima Neto et al. (2008b). The numbers (1) to (15) correspond to the experimental conditions shown in Table 1. The normalized radial profiles of $w / u_{o}$ cover a range of $z / d$ from about 30 to 120 . Dashed lines indicate model simulations using the correlations of Clift et al. (1978) to estimate the terminal velocity for individual bubbles [instead of Eq. (7)]. 
Note that the normalized radial profiles of $w / u_{o}$ cover a range of $z / d$ from about 30 to 120 . These also give credence to the model and reinforce the idea that bubbly jets behave as self-similar shear flows following Gaussian profiles not only for liquid velocity and void fraction (Figures 2 and 3), but also for bubble velocity (Figure 4). It is important to mention that model simulations using Eq. (8) to obtain bubble diameter and then the correlations of Clift et al. (1978) to estimate the terminal velocity for individual bubbles [instead of Eq. (7)] resulted in higher deviations (up to about $40 \%$ ) from the experimental results shown in Figure 4, even readjusting the parameters $\alpha, \gamma$ and $\lambda$ for a better curve fitting. This suggests that bubble swarms rise faster than individual bubbles due to drag reduction, as pointed out by Lima Neto et al. (2008b). Larger terminal velocity than individual particle velocity has also been observed in slurry jets (Zhang et al., 2011).

Figure 5 indicates that model simulations also compared well with the experimental data of liquid velocity given by Sun and Faeth (1986a,b) for dilute bubbly jets. The standard deviations were up to about $20 \%$ and the coefficient of determination was higher than 0.98 for all the cases, including tests with densimetric Froude numbers ranging from 26.4 to 47.4 (see Expt. 16-18 in Table 1). Note that the normalized axial distributions of $u_{c} / u_{o}$ cover a range of $z / d$ from about 0 to 60 .

Model simulations shown in Figure 6 also compared well with the experimental data of bubble velocity reported by Sun and Faeth $(1986 \mathrm{a}, \mathrm{b})$ for dilute bubbly jets. The standard deviations of $w_{c} / u_{o}$ were up to about $15 \%$ and the coefficient of determination was higher than 0.99 for all the tests (see Expt. 16-18 in Table 1). Model simulations using Eq. (8) and the correlations of Clift et al. (1978) to estimate the terminal bubble velocity [instead of Eq. (7)] resulted in approximately the same deviations $( \pm 15 \%)$ from the experimental results shown in Figure 6. This indicates that Eq. (7) may also be used for modeling the flow structure of dilute bubbly jets.

Figure 7 shows another validation of the present model, in which simulated results are compared with experimental data of liquid velocity given by Iguchi et al. (1997) for confined bubbly jets. The standard deviations were lower than $10 \%$ and the coefficient of determination was higher than 0.97 for all the cases, which included tests with densimetric Froude numbers ranging from 22.1 to 63.7 (see Expt. 19-21 in Table 1) and normalized axial distributions of $u_{c} / u_{o}$ covering a range of $z / d$ from about 20 to 60 .
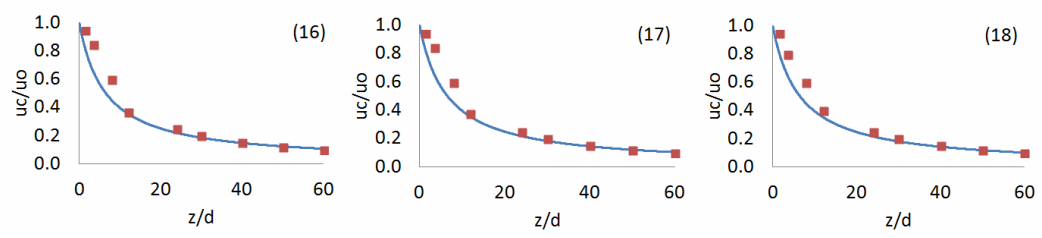

Figure 5: Comparison between model predictions and experimental data of liquid velocity given by Sun and Faeth (1986a). The numbers (16) to (18) correspond to the experimental conditions shown in Table 1.
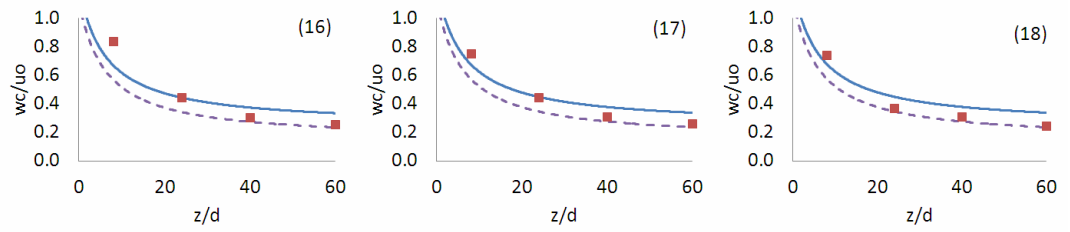

Figure 6: Comparison between model predictions and experimental data of bubble velocity given by Sun and Faeth (1986a). The numbers (16) to (18) correspond to the experimental conditions shown in Table 1. Dashed lines indicate model simulations using the correlations of Clift et al. (1978) to estimate the terminal velocity for individual bubbles [instead of Eq. (7)].
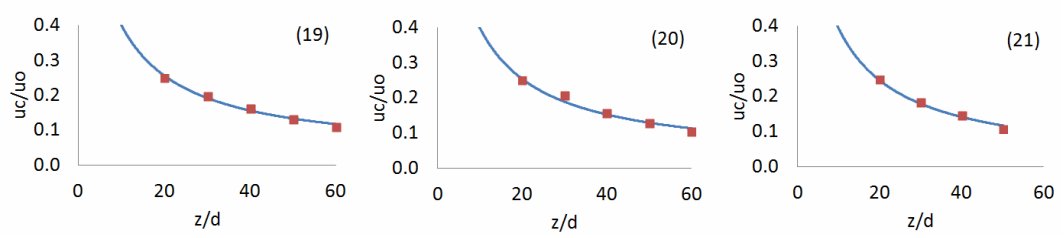

Figure 7: Comparison between model predictions and experimental data of liquid velocity given by Iguchi et al. (1997). The numbers (19) to (21) correspond to the experimental conditions shown in Table 1. 
In Figure 8, the present model simulations of centerline liquid velocity, jet radius and centerline void fraction are compared to analytical solutions based on Gaussian type self-similarity equations for singlephase buoyant jets reported by Zhang et al. (2011) and empirical correlations for bubbly jets given by Iguchi et al. (1997). The conditions for the bubbly jet simulation using the model proposed herein included: $H=1.0 \mathrm{~m}, d=5 \mathrm{~mm}, Q_{g o}=Q_{l o}=3.0 \mathrm{~L} / \mathrm{min}$, $C_{o}=50 \%, R e=12732$, and $F r=26.3$. The same value of $\mathrm{Fr}$ was used for the single-phase buoyant jet simulations, while the same value of $C_{o}$ was used for the bubbly jet simulations using the simple correlations of Iguchi et al. (1997). Note that Iguchi et al. (1997) only reported correlations for centerline liquid velocity and jet radius.

Figure 8(a) shows the decay of the normalized centerline liquid velocity $u_{c} / u_{o}$ as a function of the normalized distance from the source $z / d$. It can be seen that bubbly jets present a decay of $u_{c} / u_{o}$ with $z / d$ following a slope $\left[u_{c} / u_{o} \sim(z / d)^{-3 / 4}\right]$ that is very close to that of single-phase buoyant jets. This is consistent with the results presented before, in which the entrainment coefficient of bubbly jets $(\alpha=0.077)$ is slightly higher than that estimated herein for singlephase buoyant jets $(\alpha=0.075)$. Thus, bubbly jets and single-phase buoyant jets with the same value of $\mathrm{Fr}$ are expected to present approximately the same decay of $u_{c} / u_{o}$ with $z / d$. On the other hand, the empirical correlation of Iguchi et al. (1997) obtained for confined bubbly jets provided a steeper slope $\left[u_{c} / u_{o} \sim\right.$ $\left.(z / d)^{-1}\right]$. This suggests that the relatively small water levels in the experiments of Iguchi et al. (1997) (see Table 1) caused an earlier decay of the axial liquid velocity, as close to the free surface the jets are expected to bend radially (see Lima Neto et al., 2008a,c). But it is relevant to highlight that, for $z / d<50$ [see Figure 8(a)], the simulations using the present model and the empirical correlation of Iguchi et al. (1997) give close results, which is consistent with the results shown in Figure 7. In addition, as a secondorder effect, confinement may result in higher turbulence levels and, as a consequence, in lower mean liquid velocities along the jet centerline. In fact, the present model simulations for higher momentum amplification factors $(\gamma>1.0)$ resulted in steeper slopes than the above-mentioned value ( $>-3 / 4)$. Similarly, model simulations for higher entrainment coefficients $(\alpha>0.077)$ also resulted in steeper slopes.

Figure 8(b) shows the increase in the normalized radius $b / d$ as a function of $z / d$. Observe that for bubbly jets the radius at the nozzle exit was taken as equal to the nozzle diameter, while no correction for the virtual origin of the flow was considered for the cases of single-phase buoyant jets and confined bubbly jets. It is seen that unconfined bubbly jets spread approximately linearly with $z / d$, as do single-phase buoyant jets and confined bubbly jets. However, unconfined bubbly jets spread faster $(d b / d z \sim 0.14)$ than single-phase buoyant jets $(d b / d z \sim 0.12)$ and confined bubby jets $(d b / d z \sim 0.13)$. Such results imply that bubble-liquid interactions may play a relevant role in increasing the spreading rate of bubbly jets, as compared to single-phase buoyant jets. On the other hand, the slightly lower spreading rate of confined bubbly jets as compared to unconfined bubbly jets suggests that lateral confinement may restrict the spreading of such flows. Consistently, the abovementioned spreading rate $(d b / d z \sim 0.14)$ increased by increasing the entrainment coefficient $(\alpha>0.077)$ and the momentum amplification factor $(\gamma>1.0)$.

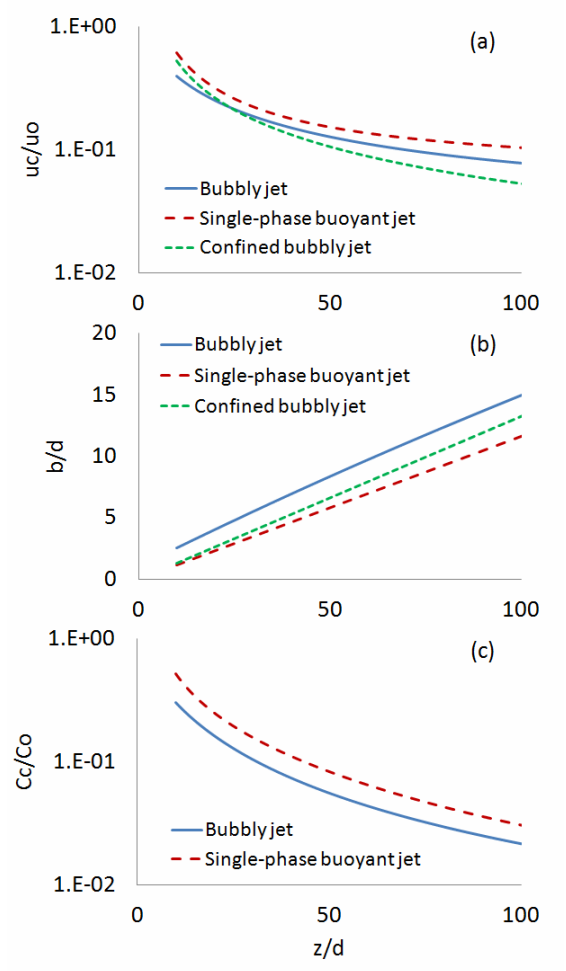

Figure 8: Simulations using the present model (bubbly jet), analytical solutions reported by Zhang et al. (2011) (single-phase buoyant jet), and empirical correlations given by Iguchi et al. (1997) (confined bubbly jet): (a) normalized centerline liquid velocity, (b) normalized radius, and (c) normalized centerline void fraction (or concentration, in the case of the single-phase buoyant jet). 
Figure $8(\mathrm{c})$ shows the decay of the normalized centerline void fraction $C_{c} / C_{o}$ (or concentration, in the case of single-phase buoyant jets) as a function of $z / d$. Again, bubbly jets present a decay of $C_{c} / C_{o}$ with $z / d$ following a slope $\left[C_{c} / C_{o} \sim(z / d)^{-5 / 4}\right]$ which is very close to that of single-phase buoyant jets. Thus, bubbly jets and single-phase buoyant jets with the same values of $\mathrm{Fr}$ are expected to present a similar decay of $C_{c} / C_{o}$ with $z / d$. Interestingly, $C_{c} / C_{o}$ decays faster than $u_{c} / u_{o}$ [see Figure 8(a)]. Model simulations for higher entrainment coefficients $(\alpha>0.077)$ and momentum amplification factors $(\gamma>1.0)$ also resulted in steeper slopes for the decay of $C_{c} / C_{o}$ than the above-mentioned value ( $>-5 / 4)$.

In Figure 9, the present model simulations of centerline bubble velocity, jet radius and centerline void fraction are compared to empirical correlations for slurry jets reported by Zhang et al. (2011). The following conditions were used for the bubbly jet simulation: $H=1.0 \mathrm{~m}, d=9 \mathrm{~mm}, Q_{g o}=2.01 / \mathrm{min}$, $Q_{l o}=4.0 \mathrm{l} / \mathrm{min}, C_{o}=33.3 \%, R e=9431$, and $F r=4.8$. The same value of $F r$ was used for the slurry jet simulations. Note that here the definition of $\mathrm{Fr}$ was modified in order to apply the slurry jet correlations (obtained for $2<F r<6$ ). Hence, for slurry jets, the term $\left(\rho_{l}-\rho_{m}\right)$ in Eq. (16) was replaced by $\left(\rho_{s}-\rho_{l}\right)$, in which $\rho_{s}$ is the sand density, while for bubbly jets, $\rho_{m}$ was replaced by $\rho_{g}$.

Figure 9(a) shows the decay of the normalized centerline bubble (or sand) velocity $w_{c} / u_{o}$ as a function of $z / d$. Bubbly jets present a decay of $w_{c} / u_{o}$ with $z / d$ following a slope $\left[u_{c} / u_{o} \sim(z / d)^{-1 / 4}\right]$ that is approximately the same of slurry jets. This implies that the buoyancy effects caused by the bubbles and sand particles on the decay of $w_{c} / u_{o}$ are similar. Thus, bubbly jets and slurry jets with the same $F r$ are expected to present a similar decay of $w_{c} / u_{o}$ with $z / d$. It is also interesting to observe that, as expected, $w_{c} / u_{o}$ decays much slower than $u_{c} / u_{o}$ [see Figure 8(a)].

Figure 9(b) shows the increase in the normalized radius $b / d$ as a function of $z / d$. Observe that no correction for the virtual origin of the flow was considered for the slurry jets. It can be seen that bubbly jets spread faster $(d b / d z \sim 0.13)$ than slurry jets $(d b / d z \sim$ 0.10 ). This probably occurs because bubble-liquid interactions play a more relevant role in increasing the spreading rate than particle-liquid interactions. In fact, bubbles are larger than sand particles and their effect is expected to be stronger. Note that $d b / d z$ for the present bubbly jet case is slightly lower than that shown in Figure 8(b).
Figure 9(c) shows the decay of the normalized centerline void fraction $C_{c} / C_{o}$ (or sand concentration, in the case of slurry jets) as a function of $z / d$. Bubbly jets present a decay of $C_{c} / C_{o}$ with $z / d$ following a slope $\left[C_{c} / C_{o} \sim(z / d)^{-5 / 4}\right]$ that is close to that of slurry jets. Hence, bubbly jets and slurry jets with the same $F r$ are expected to present a similar decay of $C_{c} / C_{o}$ with $z / d$. The results also confirm that $C_{c} / C_{o}$ decays much faster than $w_{c} / u_{o}$ [see Figure 9(a)].

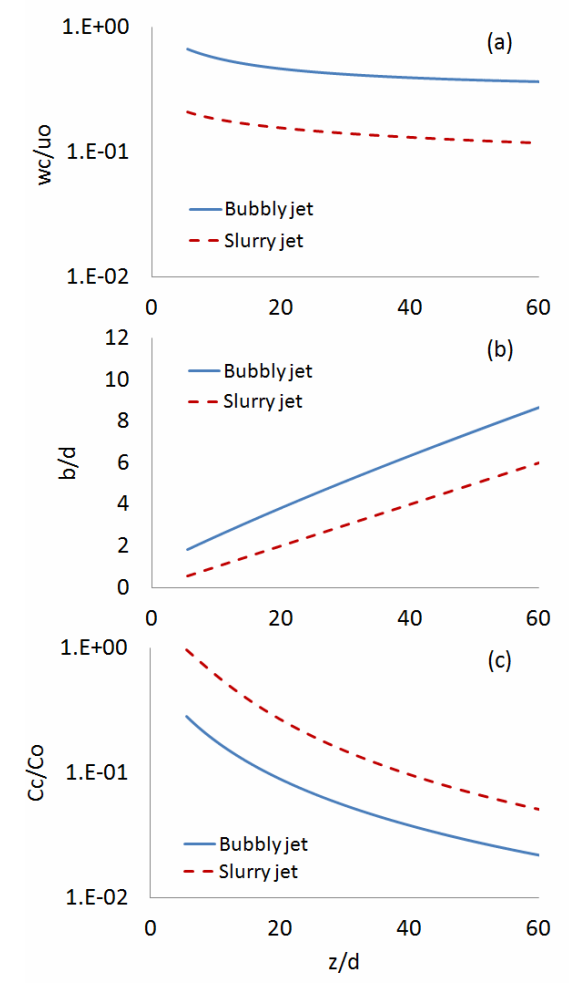

Figure 9: Simulations using the present model (bubbly jet) and empirical correlations given by Zhang et al. (2011) (slurry jet): (a) normalized centerline bubble (or sand) velocity, (b) normalized radius, and (c) normalized centerline void fraction (or concentration, in the case of the slurry jet).

\section{CONCLUSIONS}

In this article, an integral model based on the Gaussian type self-similarity was proposed to predict the axial and radial distributions of mean liquid velocity, bubble velocity and void fraction in vertical bubbly jets with nearly monodisperse bubble sizes. The model consists of a system of conservation equations for volume, momentum, and buoyancy fluxes in the form of ordinary differential equations, which were solved numerically. Adjusting the model to experimental data available in the literature for a wide range of densimetric Froude numbers provided opti- 
mized values for the entrainment coefficient $(\alpha=$ $0.077)$, momentum amplification factor $(\gamma=1.0)$, and spreading ratio of the bubble core $(\lambda=0.6)$ for different flow conditions. This resulted in standard deviations of 10 and $18 \%$ between model predictions and experimental data of liquid velocity and void fraction, respectively. The model was also validated with additional data obtained from different experimental setups, which yielded deviations between model predictions and experimental data for mean liquid velocity, bubble velocity and void fraction of up to about $20 \%$. This suggests that bubbly jets tend to behave as self-preserving shear flows.

Model predictions of liquid velocity and void fraction were very sensitive to $\alpha$. On the other hand, $\lambda$ significantly affected model predictions of void fraction, while $\gamma$ presented a small effect on liquid velocity. It was found that bubbly jets induce slightly higher entrainment $(\alpha=0.077)$ than single-phase buoyant jets $(\alpha=0.075)$, probably because of the additional entrainment into the wakes of the bubbles. The low value $(\gamma=1.0)$ fitted for the momentum amplification factor was consistent with experimental results available in the literature for single-phase jets and confined bubbly jets, which implies that the effect of turbulence on the mean flow structure of bubbly jets is of second order. The fitted value $(\lambda=$ $0.6)$ for the spreading ratio of the bubble core was smaller than that obtained in previous bubbly jet studies, but within the ranges reported in the literature for bubble plumes. In fact, a lower value of $\lambda$ was necessary in the present study in order to fit the radial profiles of void fraction.

The impact of different approaches for evaluation of the liquid velocity at the nozzle exit was also investigated, and the drift flux model provided the best results. Moreover, model comparisons with experimental data showed that the use of correlations to describe the increased slip velocity in bubble swarms provided better results than using the classical terminal velocity of individual bubbles. This reinforces previous experimental evidence that bubble swarms rise faster than individual bubbles due to drag reduction.

The present model simulations of the axial decay of centerline liquid velocity, bubble velocity, and void fraction (or concentration) indicated a behavior very similar to those of single-phase buoyant jets and slurry jets with the same densimetric Froude numbers. Nonetheless, unconfined bubbly jets presented a slower decay of liquid velocity than confined bubbly jets, probably because of the earlier bend of the liquid flow to the radial direction (close to the free surface) and the higher turbulence levels expected due to confinement. On the other hand, bubbly jets presented a higher spreading rate than single-phase buoyant jets and slurry jets, which was attributed to bubble-liquid interactions. Lateral confinement also affected the behavior of bubbly jets by lowering their spreading rates, as compared to unconfined bubbly jets.

Although some attempts have been made previously by other researchers to model the two-phase flow structure in bubbly jets using computational fluid dynamics (CFD), the agreement between measured and predicted flow characteristics is still qualitative, especially for higher gas volume fractions. Therefore, the proposed integral model, which is much simpler than CFD models, has the ability to predict the two-phase flow structure in vertical bubbly jets with gas volume fractions ranging from very low to high. This allows its application in many engineering situations involving vertical bubbly jets in reactors, tanks and water bodies. Moreover, this model clearly shows that vertical bubbly jets behave as self-preserving flows, similarly to single-phase jets and plumes.

\section{ACKNOWLEDGMENTS}

This work has been financially supported by the National Council for Scientific and Technological Development - CNPq (Project 476430/2011-9).

\section{REFERENCES}

Carazzo, G., Kaminski, E. and Tait, S., The route to self-similarity in turbulent jets and plumes. Journal of Fluid Mechanics, 547, 137-148 (2006).

Cederwall, K. and Ditmars, J. D., Analysis of airbubble plumes. KH-R-24, W. M. Keck Laboratory of Hydraulics and Water Resources, Division of Engineering and Applied Science, California Institute of Technology, Pasadena, CA (1970).

Clift, R., Grace, J. R. and Weber, M. E., Bubbles, drops and particles. Academic Press, New York (1978).

Iguchi, M., Okita, K., Nakatani, T. and Kasai, N., Structure of turbulent round bubbling jet generated by premixed gas and liquid injection. Int. J. Multiphase Flow, 23(2), 249-262 (1997).

Lima Neto, I. E., Bubble plume modelling with new functional relationships. Journal of Hydraulic Research, 50(1), 134-137 (2012a).

Lima Neto, I. E., Modeling the liquid volume flux in bubbly jets using a simple integral approach. Jour- 
nal of Hydraulic Engineering, 138(2), 210-215 (2012b).

Lima Neto, I. E., Zhu, D. Z. and Rajaratnam, N., Air injection in water with different nozzles. Journal of Environmental Engineering, 134(4), 283-294 (2008a).

Lima Neto, I. E., Zhu, D. Z. and Rajaratnam, N., Bubbly jets in stagnant water. Int. J. Multiphase Flow, 34(12), 1130-1141 (2008b).

Lima Neto, I. E., Zhu, D. Z. and Rajaratnam, N., Effect of tank size and geometry on the flow induced by circular bubble plumes and water jets. Journal of Hydraulic Engineering, 134(6), 833842 (2008c).

Lima Neto, I. E., Zhu, D. Z. and Rajaratnam, N., Horizontal injection of gas-liquid mixtures in a water tank. Journal of Hydraulic Engineering, 134(12), 1722-1731 (2008d).

Lima Neto, I. E., Zhu, D. Z., Rajaratnam, N., Yu, T., Spafford, M. and McEachern, P., Dissolved oxygen downstream of an effluent outfall in an icecovered river: Natural and artificial aeration. Journal of Environmental Engineering, 133(11), 10511060 (2007).

Milenkovic, R. Z., Sigg, B. and Yadigaroglu, G., Bubble clustering and trapping in large vortices. Part 1: Triggered bubbly jets investigated by phaseaveraging. International Journal of Multiphase Flow, 33, 1088-1110 (2007).

Milgram, H. J., Mean flow in round bubble plumes. J. Fluid. Mech., 133, 345-376 (1983).

Morchain, J., Moranges, C. and Fonade, C., CFD modelling of a two-phase jet aerator under influence of a crossflow. Water Res., 34(3), 3460-3472 (2000).

Morton, B. R., Taylor, G. I. and Turner, J. S., Turbulent gravitational convection from maintained and instantaneous sources. Proc. R. Soc. London, Ser. A, 234, 1-23 (1956).

Norman, T. L. and Revankar, S. T., Buoyant jet and two-phase jet-plume modeling for application to large water pools. Nuclear Engineering and Design, 241, 1667-1700 (2011).

Socolofsky, S. A., Bhaumik, T. and Seol, D. G., Double-plume integral models for near-field mixing in multiphase plumes. J. Hydraul. Eng., 134(6), 772-783 (2008).

Socolofsky, S. A., Crounse, B. C. and Adams, E. E., Multi-phase plumes in uniform, stratified, and flowing environments. Environmental Fluid Mechanics Theories and Applications, H. Shen, A. Cheng, K.-H. Wang, M. H. Teng, and C. Liu, Eds., ASCE, Reston (2002).

Sun, T. Y. and Faeth, G. M., Structure of turbulent bubbly jets - I. Methods and centerline properties. Int. J. Multiphase Flow, 12(1), 99-114 (1986a).

Sun, T. Y. and Faeth, G. M., Structure of turbulent bubbly jets - II. Phase properties profiles. Int. J. Multiphase Flow, 12(1), 115-126 (1986b).

Suñol, F. and González-Cinca, R., Opposed bubbly jets at different impact angles: Jet structure and bubble properties. Int. J. Multiphase Flow, 36, 682-689 (2010).

Varley, J., Submerged gas-liquid jets: Bubble size prediction. Chem. Eng. Sci., 50(5), 901-905 (1995).

Wüest, A., Brooks, N. H. and Imboden, D. M., Bubble plume modelling for lake restoration. Water Resour. Res., 28(12), 3235-3250 (1992).

Wygnanski, I. and Fiedler, H., Some measurements in the self-preserving jet. J. Fluid. Mech., 38, 577-612 (1969).

Zhang, W. M., Rajaratnam, N. and Zhu, D. Z., Transport with Jets and Plumes of Chemicals in the Environment. Encyclopedia of Sustainability Science and Technology, Robert A. Meyers (Ed.), Springer, New York (2011).

Zhang, W. M. and Zhu, D. Z., Bubble characteristics of air-water bubbly jets in crossflow. Int. J. Multiphase Flow, 55(10), 156-171 (2013).

Zuber, N. and Findlay, J. A., Average volumetric concentration in two-phase flow systems. J. Heat Transfer. 87(4), 453-468 (1965). 